\title{
A Novel Situation Awareness Model for Network Systems' Security
}

\author{
Guosheng Zhao ${ }^{1,2}$, Huiqiang Wang ${ }^{1}$, Jian Wang ${ }^{1}$, and Linshan Shen ${ }^{1}$ \\ ${ }^{1}$ Institute of Computer Science and Technology, Harbin Engineer \\ University, Harbin 150001, China \\ ${ }^{2}$ Center of Computer Network and Information, Harbin Normal \\ University, Harbin 150001, China \\ gs. zhao@yahoo.com. ch
}

\begin{abstract}
Building and maintaining the information superiority is the basis of the principle of active defense, integrated guarding in the cyberspace. In this paper, a novel method based on grey verhulst model was introduced to forecast the network system's security situation. Starting with unequal interval original risk data series, the proposed method choosed grey verhulst model or its inverse function to forecast the future risk value of network system, ant then it could modify the forecasting precision based on multilevel residual error. Simulation results reveal that the presented model not only gains the intuitionistic curve graph of network security situation, but also can achieve satisfactory precision. At the same time, it is simple in use and deserves further study to fully explore its potential for evaluation issues.
\end{abstract}

Keywords: non-equal interval, grey verhulst model, residual error revision, situation awareness.

\section{Introduction}

The situation awareness[1,2] is to analyze or comprehend various elements' behavior in time and space in active or autonomic way, as well as its evolutive tendency, attempt and prospective developments situation. It is an indispensably credible technology for safe precaution system.

Because the influencing factors of network security is complex and uncertain, so it is difficult to construct an appropriate forecasting model for security situation of network system. At present the various proposed frames[2,3,4] of cyberspace situation awareness cannot mostly provide practically useful situation information, cannot help manager make the right decision-making in the system actual security condition. According to the theory of grey system pioneered by Professor Deng[5], a system that lacks adequate information can be considered as a grey system. In this case, the network system can be view as a grey system. Considering the non-linearity of the risk data sequence, the grey verhulst model is selected and employed, which may discover the system's developmental principles and may scientifically make the quantitative forecast for future system's security situation. The verhulst model is 
suitable to model for non-monotonous swings sequence or the saturated ' $\mathrm{S}$ ' shape sequence very much, just as figure 1 demonstrated. This kind of curves can be divided into three segments as a whole: in I segment, the rate of curve slow increases; in II segment, the rate of curve is close to the linearity; in III segment, the rate of curve tends to steady value. Due to the original sequence is generally non-equal interval, so this paper chooses the non-equal interval grey verhulst model with residual error revision to carry out the modeling of the network system's security situation.

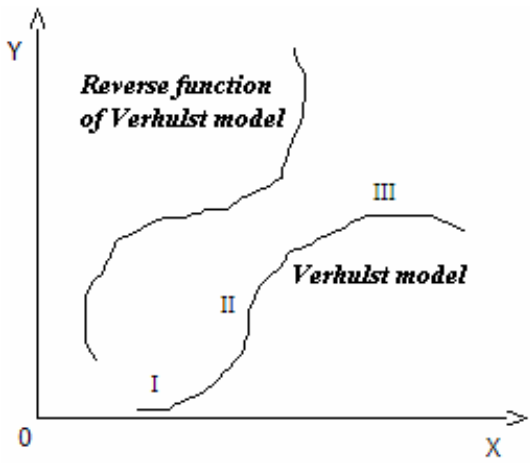

Fig. 1. The curves of verhulst model and its reverse function

This paper proposes a first-time application of mathematics based grey verhulst model for the forecasting of network system's security situation. Following contents are arranged as follows: section 1, 2 introduced the proposed model and its residual error revision method in detail; section 3 validates the model through simulation experiments; section 4 was a conclusion, which pointed out the future possible studies.

\section{Modeling Process}

\subsection{The Processing Method of Non-equal Interval Data Sequence}

Referring to the literature's processing method[6], we suppose the original sampling risk data sequence is $X^{(0)}=\left\{x^{(0)}\left(t_{k}\right), k=1,2, \ldots, n\right\}$, where $t_{i+1}-t_{i}>0$. Due to the data sequence $t_{k}$ is non-equal interval, and the difference $d t=t_{k+1}-t_{k}$ is not bigger, we may use the least squares method to construct the equal interval data sequence $t^{\prime}{ }_{k}$.According to the equation $t^{\prime}{ }_{k}=m_{0}+m_{1} \cdot k$, we can obtain the group of equations $T^{\prime}=A M$, where $A^{T}=\left(\begin{array}{cccc}1 & 1 & \cdots & 1 \\ 1 & 2 & \cdots & n\end{array}\right), \quad M=\left(\begin{array}{c}m_{0} \\ m_{1}\end{array}\right)$.In order to make $(d t)^{T} d t$ minimum, let $\frac{\partial(d t)^{T} d t}{\partial M}=0$, thus we can deduce $A^{T} A M-A^{T} T=0$, namely,

$$
M=\left(A^{T} A\right)^{-1} A^{T} T
$$


Then, we can gain $T^{\prime}$ using $t^{\prime}{ }_{k}=m_{0}+m_{1} k$.Finally, we uses Langrange method to obtain the equal interval data sequence $X^{(0)}\left(T^{\prime}\right)$ again.

\subsection{The Establishment of Non-equal Interval Verhulst Model}

After the processing of equal interval, we can obtain equal interval data sequence, and then we may use the verhulst model to carry out modeling and forecasting. Let the original data sequence be $X^{(0)}=\left\{x^{(0)}(t) \mid t=1,2, \ldots, n\right\}$, then the $A G O$ (accumulated generation operation) of $X^{(0)}$ is $X^{(1)}=\left\{x^{(1)}(t) \mid t=1,2, \ldots, n\right\}$, where $x^{(1)}(t)=\sum_{i=1}^{k} x^{(0)}(i)$, $t=1,2, \ldots, n$, and let $x^{(1)}(1)=x^{(0)}(1)$, the nonlinear differential equation of $X^{(1)}(t)$ can be synthesized as follows:

$$
\frac{d X^{(1)}(t)}{d t}=a X^{(1)}(t)-b\left(X^{(1)}(t)\right)^{2}
$$

where $a$ denotes the development coefficient, whose size and sign reflect the development situation of $X^{(0)} ; b$ denotes the system's input and its value presents some kind of grey information. These coefficients $a, b$ can be identified by the least squares method as follows:

$$
[a, b]^{T}=\left[B^{T} B\right]^{-1} B^{T} Y
$$

where $B=\left(\begin{array}{cc}\frac{1}{2}\left(x^{(1)}(1)+x^{(1)}(2)\right) & -\frac{1}{4}\left(x^{(1)}(1)+x^{(1)}(2)\right)^{2} \\ \frac{1}{2}\left(x^{(1)}(2)+x^{(1)}(3)\right) & -\frac{1}{4}\left(x^{(1)}(2)+x^{(1)}(3)\right)^{2} \\ \vdots & \vdots \\ \frac{1}{2}\left(x^{(1)}(n-1)+x^{(1)}(n)\right) & -\frac{1}{4}\left(x^{(1)}(n-1)+x^{(1)}(n)\right)^{2}\end{array}\right), Y_{N}=\left[x^{(0)}(2), x^{(0)}(3), \ldots, x^{(0)}(n)\right]^{T}$.

Consequently, from Eq. (2), $\hat{X}^{(1)}(t)$ can be observed as

$$
\hat{X}^{(1)}(t)=\frac{\frac{a}{b}}{1+\left(\frac{a}{b} \cdot \frac{1}{X^{(0)}(1)}-1\right) e^{-a(t-1)}}
$$

Furthermore, from Eq. (4), can acquire the forecasted value as

$$
\hat{X}^{(0)}(t)=\hat{X}^{(1)}(t)-\hat{X}^{(1)}(t-1)
$$

Thus, the above equation from Eq. (2-5) is the non-equal interval Verhulst model.

\subsection{Inverse Function Model of Grey Verhulst Model}

The actual risk-time curve of network security situation frequently takes on the shape of reverse "S", which is similar to the curve of Verhulst model's inverse function. This kind of curves can be also divided into three segments as a whole: in I segment, 
the rate of curve linearly increases; in II segment, the rate of curve slow grows; in III segment, the movements fast goes up, and finally tend to peak value. Therefore, we may use the inverse function model of non-equal interval grey verhulst model to analyze the reverse " $S$ " characters of security situation curve.

Through solving Eq. (4) for a reverse function solution of $\hat{X}^{(1)}(t)$, we obtain the following inverse function model of grey verhulst:

$$
\hat{X}^{(1)}(t)=\frac{1}{a} \ln \frac{\left(a-b t_{0}\right) t}{a t_{0}-b t_{0} t}+\hat{X}^{(0)}(t)
$$

In above equation, $t$ denotes a time: a, b represents the coefficient of expression. Thus, using the Eq. (5) to regress, we may obtain the forecast value of the original data sequence.

\section{Model's Residual Error Revision}

Definition 1. Suppose $\mathcal{E}^{(0)}=\left(\mathcal{E}^{(0)}(1), \mathcal{E}^{(0)}(2), \ldots, \mathcal{E}^{(0)}(n)\right)$ is the residual error sequence, where $\varepsilon^{(0)}(k)=x^{(1)}(k)-\hat{x}^{(1)}(k)$ is the residual error sequence of $X^{(1)}$. If $\exists k_{0}$, make $\varepsilon^{(0)}\left(k_{0}\right)<0$, where $\forall k, k \neq k_{0}$, has $\varepsilon^{(0)}\left(k_{0}\right) \leq \mathcal{E}^{(0)}(k)$, and then let $E$ be constant, make $E>\left|\varepsilon^{(0)}\left(k_{0}\right)\right|$, call $\left(\varepsilon^{(0)}(1)+E, \varepsilon^{(0)}(2)+E, \ldots, \mathcal{E}^{(0)}(n)+E\right)$ be the modeling residual error sequence, still marks $\left(\varepsilon^{(0)}(1), \mathcal{E}^{(0)}(2), \ldots, \mathcal{E}^{(0)}(n)\right)$.

Definition 2. Suppose the residual error sequence $\mathcal{E}^{(0)}$ is the original data sequence's one-order $A G O$ sequence, then the time response expression of grey GM $(1,1)$ model can be obtained.

$$
\hat{\varepsilon}^{(1)}(k+1)=\left(\varepsilon^{(0)}\left(k_{0}\right)-\frac{b_{\varepsilon}}{a_{\varepsilon}}\right) \exp \left[-a_{\varepsilon}\left(k-k_{0}\right)\right]+\frac{b_{\varepsilon}}{a_{\varepsilon}}, \quad k \geq k_{0}
$$

Let $\hat{e}^{(0)}=\left(\hat{\varepsilon}^{(0)}\left(k_{0}\right), \hat{\varepsilon}^{(0)}\left(k_{0}+1\right), \ldots, \hat{\varepsilon}^{(0)}(n)\right)$ as the simulation sequence of $\hat{\mathcal{E}}^{(0)}$, where $\hat{\varepsilon}^{(0)}(k+1)=\left(-a_{\varepsilon}\right)\left(\varepsilon^{(0)}\left(k_{0}\right)-\frac{b_{\varepsilon}}{a_{\varepsilon}}\right) \exp \left[-a_{\varepsilon}\left(k-k_{0}\right)\right]-E, \quad k \geq k_{0}$.

Definition 3. If we revise $\hat{X}^{(1)}$ with $\hat{\varepsilon}^{(0)}$, then the time response of expression amended is:

$$
\hat{X}^{(1)}(k+1)=\left\{\begin{array}{c}
\frac{\frac{a}{b}}{1+\left(\frac{a}{b} \cdot \frac{1}{X^{(0)}(1)}-1\right) \mathrm{e}^{-a(t-1)}}, \mathcal{E}^{(0)}(k) \notin \mathcal{E}^{(0)} \\
\frac{\frac{a}{b}}{1+\left(\frac{a}{b} \cdot \frac{1}{X^{(0)}(1)}-1\right) \mathrm{e}^{-a(t-1)}}+\hat{\mathcal{E}}^{(0)}(k+1), \mathcal{E}^{(0)}(k) \in \mathcal{E}^{(0)}
\end{array}\right.
$$

we call the Eq. (8) as the residual error revision model of grey verhulst. 
If the forecasted precision has not been good once, we may also establish the multilevel residual error revision. The so-called multilevel residual error is the difference of the forecast value and the original accumulation value after many times revising. For instance, if the forecasted value revised $\mathrm{k}$ times is $\hat{X}^{(1)}(t, k)$, the $\mathrm{kth}$ times residual error is $\varepsilon^{(0)}(t, k)$, where $\varepsilon^{(0)}(t, k)=x^{(1)}(t)-\hat{x}^{(1)}(t, k)$ and $\hat{x}^{(1)}(k, 0)=\hat{x}^{(1)}(k)$, we thus have $\hat{x}^{(1)}(t, k)=\hat{x}^{(1)}(t, 0)+\sum_{i=0}^{k-1} \varepsilon^{(0)}(t, i)$.

A model can't be applied to predict, unless it is tested well. Generally, when we predict, whether the value of prediction and actual value is consistent or not is more important than the predicted value itself. Here, we uses the backward error-detection test method[7] to carry out the measure, furthermore the model's precision can be depicted together by backward error-detection ratio $C$ and small error probability $P$ (see Table 1).

Table 1. The test standard of model precision

\begin{tabular}{ccccc}
\hline $\begin{array}{l}\text { Forecasting } \\
\text { Precision }\end{array}$ & Good & Qualification & $\begin{array}{l}\text { Reluctant } \\
\text { Qualification }\end{array}$ & Disqualification \\
\hline P & $>0.95$ & $0.95-0.80$ & $0.80-0.70$ & $<0.70$ \\
\hline C & $<0.35$ & $0.35-0.50$ & $0.50-0.65$ & $>0.65$ \\
\hline
\end{tabular}

\section{Simulations}

By taking the attack data collected by network security organize - HoneyNet[8] in November as test data, we continued the tracing of security situation of network system in this month following the proposed approach. Using the method[9] to process the test data first, we can obtain the initial non-equal interval generating series shown in the following table 2, namely $X^{(0)}\left(t_{i}\right)=\{18.5,5.0,3.7,8.2,16.4,12.0,6.6,12.1\}$, where $(i=1,2, \ldots, 8), T=\{0,2,5,10,14,17,19,22\}$. After being processed, having $M=\left(\begin{array}{c}m_{0} \\ m_{1}\end{array}\right)=\left(\begin{array}{c}-3 \\ 4\end{array}\right), \quad t_{i}{ }^{\prime}=m_{0}+m_{1} \cdot i,(i=1,2, \ldots, 8)$, and the equal interval time series are $T^{\prime}=\{1,5,9,13,17,21,25,29\}$. Let $T$ and $X^{(0)}(T)$ be the raw data, we use the interpolation method to get the equal interval data series, namely, $X^{(0)}\left(T^{\prime}\right)=\{17.9,3.7,7.5,14.6,12.0,8.3,4.4,11.8\}$.

Table 2. The initialized non-equal interval generating series

\begin{tabular}{l|cccccccc}
\hline Number & 1 & 2 & 3 & 4 & 5 & 6 & 7 & 8 \\
\hline Date & $11-08$ & $11-10$ & $11-13$ & $11-18$ & $11-22$ & $11-25$ & $11-27$ & $11-30$ \\
Risk value & 18.5 & 5.0 & 3.7 & 8.2 & 16.4 & 12.0 & 6.6 & 12.1 \\
\hline
\end{tabular}


Accumulating $X^{(0)}\left(T^{\prime}\right)$ by $\mathrm{AGO}$, we can get $X^{(1)}\left(T^{\prime}\right)$, whose value is $\{17.9,21.6,29.1,43.7,55.7,64.0,68.4,80.2\}$. Because the curve slope of AGO series rapidly increase in II section, so we adopted the Verhulst model to directly modeling. Due to Eq. (2)-Eq. (6),the forecast model can be list as table 3 shown.

Table 3. The non-equal interval Verhulst model

\begin{tabular}{c|cccccccc}
\hline Number & 1 & 2 & 3 & 4 & 5 & 6 & 7 & 8 \\
\hline Date & $11-08$ & $11-10$ & $11-13$ & $11-18$ & $11-22$ & $11-25$ & $11-27$ & $11-30$ \\
$X^{(0)}$ & 17.9 & 3.7 & 7.5 & 14.6 & 12.0 & 8.3 & 4.4 & 11.8 \\
$X^{(1)}$ & 17.9 & 21.6 & 29.1 & 43.7 & 55.7 & 64.0 & 68.4 & 80.2 \\
$\hat{X}^{(1)}$ & 17.9000 & 25.0104 & 33.7789 & 43.8182 & 54.3800 & 64.5471 & 73.5311 & 80.8884 \\
$\varepsilon$ & 0.0000 & 3.4104 & 4.6789 & 0.1182 & -1.3199 & 0.5471 & 5.1311 & 0.6884 \\
\hline Model & \multicolumn{7}{|c}{$X^{(1)}(k+1)=1 /(0.01003+0.045836 * \exp (-0.42542 k))$} \\
\hline
\end{tabular}

The model's precision is good, where $C=\frac{s e}{s x}=\frac{2.2478}{21.6376}=0.1037<0.35, P=\{|\varepsilon(k)-\bar{\varepsilon}|<0.6745 s x\}=1>0.95$. However, the individual residual error is obviously bigger, we need carry out the revision of residual error. Let $\left\{\mathcal{E}^{(0)}(2), \mathcal{E}^{(0)}(3), \mathcal{E}^{(0)}(5), \mathcal{E}^{(0)}(7)\right\}$ be a raw residual error sequence, thus the time response expression of $\hat{\varepsilon}^{(1)}(k+1)$ can be obtained as $\hat{\varepsilon}^{(1)}(k+1)=56.53987 \exp (-0.075903 * k)-51.129497$. After regressed to the former model, the final results shows in table 4.

Table 4. The non-equal interval Verhulst model with residue error revision

\begin{tabular}{|c|c|c|c|c|c|c|c|c|}
\hline Number & 1 & 2 & 3 & 4 & 5 & 6 & 7 & 8 \\
\hline$X^{(1)}$ & 17.9 & 21.6 & 29.1 & 43.7 & 55.7 & 64.0 & 68.4 & 80.2 \\
\hline$\hat{X}^{(1)}$ & 17.9000 & 22.3503 & 30.7515 & 43.8182 & 50.5276 & 64.5471 & 68.7178 & 80.8884 \\
\hline$\varepsilon^{\prime}$ & 0.0000 & 0.7503 & 1.6515 & 0.1182 & 5.1724 & 0.5471 & 0.3178 & 0.6884 \\
\hline Model & & $1003+0$ & $\frac{1}{5838 *}$ & -0.425 & $\frac{}{2 *(k))}+$ & & & 1 \\
\hline
\end{tabular}

After being checked by the backward error-detection test method, the above model's precision is good and the size of each residual error is less. For $\hat{X}^{(0)}\left(T^{\prime}\right)$, it can be obtained from Eq. [7], and then let $\hat{X}^{(0)}\left(T^{\prime}\right)$ and $T^{\prime}$ be the raw data, so we can 
get $X^{(0)}(T)$ using the interpolation method. Thus, we obtained the new model with the residual error revision.

Now, we use the new model with the residual error revision and the model without the residual error revision to forecast the security situation of network system from December 1 to December 5 respectively, the sampled data is randomly choosed in different time span of every day. The curve fitting between the forecasted result and the actual value can be seen from figure 2 .

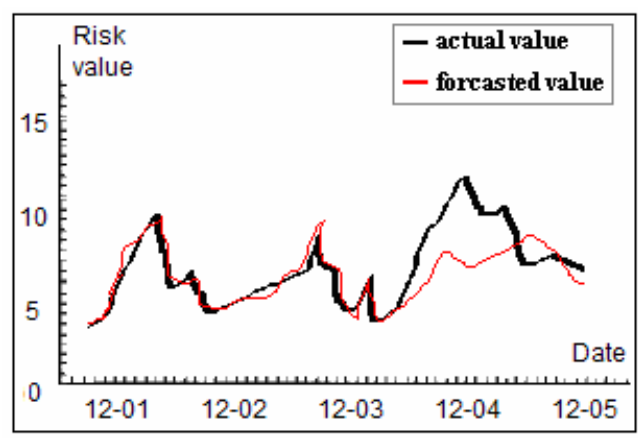

(a) The model without residual error revision

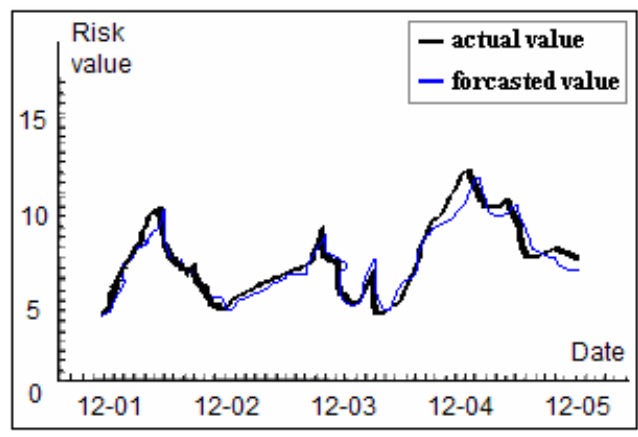

(b) The model with residual error revision

Fig. 2. The evolutive curve of network system's security situation

Seen from figure 2(a), the forecasted error is smaller during the initial three days, but along with days passing, the forecasted error has the tendency of increase and the forecasted precision gradually drop, which accords with the verhulst model itself shortcoming. Moreover, the situation curves in figure 2(b) was revised time and again, which may be obviously seen that the precision greatly enhances, the curve fitting between the forecasted result and the actual value are really satisfied. The above-mentioned results indicated that the non-equal interval grey verhulst model with residual error revision can effectively forecast the relative long-term security situation of network system. 


\section{Conclusions}

This paper proposed a non-equal interval grey verhulst model with residual error revision to predict network system security's development situation for the first time. The experimental results show the model effectively overcome the discrete traits of original data, can obtain the reasonable network security development tendency even if in uncertain or lack information, and has certain practical value. Although the proposed method has done some work in the realization aspect of network security situation awareness, there are still many important problems not been considered, such as the suitable question of massive samples, the selection way of residual error sequence, as well as model's time and spatial complexity and so on, which waits for further research.

\section{References}

1. Mark, L. H., Olga, S., Karen, A. H.: On-line situation assessment for unmanned air vehicles. Proceedings Fourteenth International Florida Artificial Intelligence Research Society Conference. Florida: Key West, FL(2001)44-48

2. Tim, B.: Intrusion systems and multisensor data fusion: creating cyberspace situational awareness. Communications of the ACM.43(4) (2000)99-105

3. Adam, X. M., Greg, L. Z., Kao, S.P.: A Computational Situation Assessment Model for Nuclear Power Plant Operations. IEEE Transactions on Systems, Man and Cybernetics. 27(6)(1997)728-742

4. Sandeep, M., Rinkus, G., Illgen, C., et al.: OLIPSA: On-line intelligent situation awareness in the tactical air processor for situation assessment.Second Annual Symposium and Exhibition on Environment. Patuxent River, MD(1997)

5. Deng, J.L.: Grey system theory .Huhan: Huazhong University of Science and Technology Press(2002)

6. Zhu, H.J.,Ma, S.J. :Application of Non-equal Interval Gray Model to Forecast of Building Subsidence. Engineering of Surveying and Mapping.10(4) (2001)39 41

7. Deng, J.L., Properties of the grey forecasting model of GM $(1,1)$, grey system, China Ocean Press, Beijing.(1988)79-90

8. Honeynet Project. Know your enemy: statistics. http://www.HoneyNet.org/papers/stats/, 2001-07-22

9. Chen, X.Z.,Zheng, Q.H.,Guan X.H.: Study on Evaluation for Security Situation of Networked Systems, Journal of Xi'an JiaoTong University.38(4)(2004)404-408 